\title{
Clonal diversity and epidemiological characteristics of ST239-MRSA strains
}

Mahmoud Bendary ${ }^{1}$, WAEL HEGAZY ${ }^{2}$, alaa sweed ${ }^{3}$, Mohamed Samir ${ }^{1}$, rasha mosbah ${ }^{3}$, Doaa Ghaith ${ }^{4}$, Ahmed Abdelaziz ${ }^{5}$, Hazem Ramadan ${ }^{6}$, Wala Alshareef ${ }^{1}$, and Marwa Abd El Hamid $^{7}$

${ }^{1}$ Affiliation not available

${ }^{2}$ Zagazig University Faculty of Pharmacy

${ }^{3}$ Zagazig University

${ }^{4}$ Cairo University

${ }^{5}$ Zagazig University Faculty of Veterinary Medicine

${ }^{6}$ Faculty of Veterinary Medicine, Mansoura University

${ }^{7}$ faculty of veterinary medicine

September 24, 2020

\begin{abstract}
Despite the wealth of knowledge available on the diversity of methicillin resistant Staphylococcus aureus (MRSA) strains that belonged to different clones, there have been no reports that analyzed such diversity on isolates belonging to the same clone. This study aimed to determine the diversity of ST239-MRSA strains, the predominant MRSA clone in worldwide, and to illustrate the correlation between the toxin genes, antimicrobial resistances and the genetic background of these strains. The molecular epidemiology of ST239-MRSA clone was analyzed by phenotypic antibiotyping, various genotypic assays. We found that coaRFLP showed the highest discriminatory power in opposition to agr typing that had the lowest discriminatory power. The majority of our isolates (80\%) were MDR, belonged to SCCmec III, agr I, coa genotype I and harbored sea and pvl genes. Our study demonstrated an overall negative correlation between the antimicrobial resistance and the toxin gene profiles. Meanwhile, there was no significant correlation between the toxin profiles and genetic background. The high diversity of ST239-MRSA strains might be among the contributors to the reported difficulties in control and prevention of MRSA infection in Egypt. With such diversity, it is assumed that these strains may have undergone different evolutionary processes.
\end{abstract}

\section{Clonal diversity and epidemiological characteristics of ST239-MRSA strains}

Mahmoud M. Bendary ${ }^{1}$ Wael A.H. Hegazy ${ }^{2}$, Alaa H. Sewid ${ }^{3}$, Mohamed Samir ${ }^{4}$, Rasha A. Mosbah ${ }^{5}$, Doaa M. Ghaith ${ }^{6}$, Ahmed S. Abdelaziz ${ }^{7}$, Hazem Ramadan ${ }^{8,9}$, Wala. A. Alshareef ${ }^{10}$ and Marwa I. Abd El-Hamid ${ }^{* 3}$

1 Department of Microbiology and Immunology, Faculty of Pharmacy, Port Said University, Port Said, 42511, Egypt

2 Department of Microbiology and Immunology, Faculty of Pharmacy, Zagazig University, Zagazig, 44511, Egypt

3 Department of Microbiology, Faculty of Veterinary Medicine, Zagazig University, Zagazig, 44511, Egypt

4 Department of Zoonoses, Faculty of Veterinary Medicine, Zagazig University, Zagazig

5 Fellow Pharmacist at Zagazig University Hospital, Zagazig, 44511, Egypt. 
6 Department of Clinical and Chemical Pathology, Faculty of Medicine, Cairo University,

University, Mansoura, 35511, Egypt

7 Department of Pharmacology, Faculty of Veterinary Medicine, Zagazig University, Zagazig, 44519, Egypt

8 Department of Hygiene and Zoonoses, Faculty of Veterinary Medicine, Mansoura, Athens, GA 30605, USA

9 Bacterial Epidemiology and Antimicrobial Resistance Research Unit, US National Poultry Research Center, USDA-ARS Cairo, 11511, Egypt

10 Department of Microbiology and Immunology, Faculty of Pharmacy, 6 October University, 6 October, 12566, Egypt

\section{*Corresponding authors:}

\section{Marwa Ibrahim Abd El-Hamid}

Assistant Professor of Microbiology

Department of Microbiology, Faculty of Veterinary Medicine, Zagazig University, Egypt

Postal address: Saleh Abo Rahil Street 19, El-Nahal, 44519, Zagazig, Sharkia, Egypt

Phone: +20 0552267009

Fax: +20552283683

Email: mero_micro2006@yahoo.com,mero_micro@zu.edu.eg

Orichid ID: 0000-0002-1560-6158

Short running head : ST239-MRSA clone

\section{Summary}

Despite the wealth of knowledge available on the diversity of methicillin resistant Staphylococcus aureus (MRSA) strains that belonged to different clones, there have been no reports that analyzed such diversity on isolates belonging to the same clone. This study aimed to determine the diversity of ST239-MRSA strains, the predominant MRSA clone in worldwide, and to illustrate the correlation between the toxin genes, antimicrobial resistances and the genetic background of these strains. The molecular epidemiology of ST239-MRSA clone was analyzed by phenotypic antibiotyping, various genotypic assays. We found thatcoa RFLP showed the highest discriminatory power in opposition toagr typing that had the lowest discriminatory power. The majority of our isolates (80\%) were MDR, belonged to SCCmec III, agr I, coa genotype I and harbored sea and $p v l$ genes. Our study demonstrated an overall negative correlation between the antimicrobial resistance and the toxin gene profiles. Meanwhile, there was no significant correlation between the toxin profiles and genetic background. The high diversity of ST239-MRSA strains might be among the contributors to the reported difficulties in control and prevention of MRSA infection in Egypt. With such diversity, it is assumed that these strains may have undergone different evolutionary processes.

Key words: MRSA; ST239; MDR; genotyping; evolution

\section{Introduction}

Methicillin-resistant Staphylococcus aureus (MRSA) has traditionally been considered a global pathogen. The ST239 is the dominant MRSA clone overtime worldwide, notably in Egypt (Abd El-Hamid et al., 2019) and has been widely reported in Asia, Europe, Middle East and South and North America (Monecke et al., 2011). In terms of genetic structure, ST239-MRSA is an interesting clone with six of the seven multi-locus sequence typing (MLST) housekeeping genes being identical to ST8. However, ST239 and its parallels ST240 and ST241 differ from ST8 clone in $\operatorname{arc} C$ allele $(\operatorname{arc} C-2$ in the formers, instead of $\operatorname{arc} C-3$ in the latter) (Robinson and Enright, 2004). 
The genetic typing is warranted to enable better understanding of the infection dynamics. Among the available typing methods, for MRSA clone in general including ST239-MRSA clone in particular are staphylococcal cassette chromosome mec (SCCmec) elements, accessory gene regulator (agr) alleles, coagulase (coa ) genotypes, restriction fragment length (RFLP) patterns of the coa gene (coa-RFLP) and the variable repeat region of $S$. aureusprotein A ( spa) gene. These methods are known to be essential for determining the origin and the clonal relations and are used for enhanced discrimination of the closely related ST239-MRSA strains(Kong et al., 2017).

The ability of ST239-MRSA clone to produce toxins and to resist certain antimicrobials affords a survival advantage and fitness under adverse conditions. Indeed, ST239-MRSA have been reported to be multidrug resistant (MDR), posing serious public health concerns(Kong et al., 2017). Another fitness feature of ST239MRSA is the ability to produce several toxins such as Panton-Valentine leucocidin ( $p v l$ ), toxic shock syndrome toxin (tst), exfoliative toxins A (eta) and B (etb) and staphylococcal enterotoxins A-G (sea -seg ), which cause harmful toxic effects to the host(Bendary et al., 2016). The correlation between the existence of toxin genes and antimicrobial resistance traits depends on many factors including the bacterial species, virulence and antimicrobial resistance, the ecological niche and the host(Beceiro et al., 2013). However, many of these questions remain unclear. Previous studies have indicated that some toxigenic strains belonged to a particular molecular type of the MRSA(Baba T, 2002). This implies that the molecular characteristics of MRSA strains affect their toxin gene profiles(Baba T, 2002).

The present study aimed to investigate the molecular epidemiology and diversity of ST239-MRSA clone belonging to different hosts and sample types using a combination of typing approaches. We also reported the associations and differences among these typing methods as well as their discriminatory abilities. The knowledge gained from this study would enable better understanding of the characteristics of ST239-MRSA clone and thus could contribute to the enhancement of its prevention and control in Egypt.

\section{Materials and methods}

This is a case series study carried out to explain the cross-section of genetic diversity among the detected ST239-MRSA isolates during the period from June 2016 to august 2019.

\section{Ethics statement}

The ethical approval of participants was not necessary since all $S$. aureus isolates were kindly provided from different microbiology laboratories in Zagazig City, Sharkia Governorate, Egypt and no clinical samples or human participants were involved in this research.

\section{Bacterial isolates}

In this study, we investigated a total of 105 S. aureus isolates from both human (60) and animal (45) sources in Zagazig City, Sharkia Governorate, Egypt, including thirty ST239- MRSA strains which were kindly provided from microbiology laboratory, Zagazig University. The human strains were recovered from different Zagazig University hospital units.

\section{Identification of $S$. aureus isolates}

The identification of all $S$. aureus isolates was based phenotypically on standard bacteriological methods (Becker, 2015) in addition to API 20S identification kit (BioMerieux, Marcy l'Etoile, France) and confirmed using PCR analyses of 16S rRNA and a species-specific nucgene(Abd El-Hamid and Bendary, 2015). For methicillin resistance analysis, oxacillin and cefoxitin antibiotic susceptibility testing and PCR detection of $m e c A$ gene were performed (Abd El-Hamid and Bendary, 2015). The sequence type (ST) was characterized by MLST, which was based on seven house-keeping genes (arc C, aro E,glp F, gmk, pta, tpi A and yqi L). Each sequence was then compared to the $S$. aureus MLST database (http://saureus.mlst.net/) to obtain an allele number as was previously described (Enright et al., 2000) .

\section{Antibiotyping assay}


Antimicrobial susceptibility of all ST239-MRSA strains was tested by disk diffusion method using the standard antimicrobial discs (Oxoid, UK) and according to the guidelines of Clinical and Laboratory Standards Institute (CLSI)(CLSI, 2014). Strains susceptibility was examined against 13 types of antimicrobials including oxacillin (OX; $1 \mu \mathrm{g}$ ), cefoxitin (FOX; $30 \mu \mathrm{g}$ ), ceftriaxone (CRO; $30 \mu \mathrm{g}$ ), imipenem (IPM; $10 \mu \mathrm{g}$ ), vancomycin (VA; $30 \mu \mathrm{g}$ ), clindamycin (DA; $2 \mu \mathrm{g}$ ), rifamycin SV (RF; $30 \mu \mathrm{g}$ ), trimethoprim-sulfamethoxazole (SXT; $1.25 / 23.75 \mu \mathrm{g})$, erythromycin (E; $15 \mu \mathrm{g})$, ciprofloxacin (CIP; $5 \mu \mathrm{g}$ ), tetracycline (TE; $30 \mu \mathrm{g}$ ), gentamicin $(\mathrm{CN} ; 10 \mu \mathrm{g})$ and chloramphenicol $(\mathrm{C} ; 30 \mu \mathrm{g})$. The minimum inhibitory concentration (MIC) of vancomycin was determined phenotypically using the broth micro dilution method(Franklin RC et al., 2012). Moreover, the vancomycin resistance was confirmed by a multiplex PCR amplification of van A and van B genes under the same thermal conditions reported previously(Kariyama et al., 2000). The multiple antibiotic resistance (MAR) indices for the examined isolates were performed as was previously described(Tambekar et al., 2006). Of note, the strains were defined as MDR if they were resistant to at least three different antimicrobial classes(Magiorakos et al., 2012).

\section{Characterization of ST239-MRSA genetic background and toxin gene profiles}

All ST239-MRSA strains were analyzed for the detection of their genomic variations using uniplex PCR assays of coa and spa genes as stated previously(Hookey et al., 1998, Larsen et al., 2008). Digestion of coa PCR products with Alu I restriction endonuclease (Sigma, USA) was then performed. Moreover, multiplex PCRs were performed for agr and SCCmec typing as was described previously(Zhang et al., 2005, Gilot et al., 2002). Characterization of toxin gene profiles by detecting sea -seg, eta, etb, tst and pvl genes were performed using the previously reported PCR conditions(Mehrotra et al., 2000). For the quality control of the genotyping methods, $S$. aureus ATCC25923 and E. coli ATCC25922 were included with all PCR runs as positive and negative controls, respectively.

\section{Bioinformatics and statistical analyses}

Simpson's index (Simpson, 1949) applied on the whole strains $(\mathrm{n}=50)$ as well as on those per each host were used. Shapiro test (Royston, 1982) and Q-Q plot (Katayama et al., 2000) were used to assess and visualize the distribution of all variables. The correlation analyses and visualization were done using $R$ packagescorrplot, heatmaply, hmisc andggpubr (Friendly, 2002, Galili et al., 2018, Jr, 2020). A heatmap supported by a hierarchical clustering dendrogram was generated to visualize the phenotype/genotype profiles of the examined isolates (Kolde, 2018). The analyses were conducted by using the R environment (v. 3.6.2) (Team, 2018).

\section{Results}

\section{Multilocus sequence typing of $S$. aureus strains}

According to the MLST typing results of 105 S.aureus isolates, ST239 was proven for 50 isolates. They were recovered from 18 milk samples of mastitis cows and 32 human patients, including 6 sputum, 6 wound swabs, 6 urine, 7 pus, 4 blood, 2 cerebrospinal fluid (CSF) and 1 pericardial fluid. The different allele sequences of the newly identified ST239- S.aureus strains in this study have been deposited in the Genbank with accession numbers of MN880894: MN881033.

\section{Antibiogram typing of ST239-MRSA clone}

Analysis of the antimicrobial resistance patterns of all ST239 S. aureus isolates $(\mathrm{n}=50)$ revealed that all of them (100\%) were uniformly resistant to oxacillin and cefoxitin and therefore, they were identified as ST239MRSA. Irrespective of the host, the highest resistance of ST239-MRSA isolates was against ciprofloxacin (54\%). Meanwhile, relatively low resistance rate was observed against vancomycin (8\%) and imipenem (4\%) (Table S1 ). Only the occurrence of clindamycin resistance was significantly different among the studied hosts $(P=0.05)$. Both human and animal isolates are alarmingly resistant to the vancomycin (MIC > $16 \mu \mathrm{g} / \mathrm{mL}$ ) with percentages of $6.25 \%$ and $11.1 \%$, respectively. Interestingly, ST239-MRSA strains were resistant to at least $2^{\sim} 9$ of the 13 antimicrobials generating MAR indices ranged from 0.15 to 0.69 ( $D$-value $=0.86)$. Among our human strains, an alarming percentage of MDR $(90.6 \%, 29 / 32)$ was observed, while 
14 out of 18 cow strains (77.8\%) were MDR. Antibiotyping of the examined strains revealed 26 different antimicrobial resistance patterns with $D$-value equal to 0.96 (Fig. 1B, Table S1 ).

\section{Correlation among resistances to different antimicrobials}

The correlation among individual antimicrobial resistance phenotypes was variable and showed an overall weak positive correlation (average $\mathrm{R}=0.1$ ). Ceftriaxone and tetracycline showed the highest positive correlation (R-value $=1, P<0.05$ ), followed by ceftriaxone/erythromycin and tetracycline/erythromycin pairs (R-values $=0.8, P<0.05)$. The clindamycin correlated negatively in a significant manner with tetracycline $($ R-value $=-0.4, P<0.05)($ Fig. 2$)$.

Strains diversity based on antimicrobial resistance profile among different hosts and sample types

Analyzing the antimicrobial resistance patterns demonstrated a high diversity of ST239-MRSA strains belonging to different hosts and from different sample types as was evidenced by their large overlap revealed by the nMDS analyses (Fig. 1A ) with averaged binary distances equating 0.4-0.5 among and within hosts (Fig. 3A ), 0.2-0.5 among and within samples (Fig. 3B ) and averaged correlation coefficient (R) of 0.1-0.2 among hosts.

The averaged binary distances and correlation coefficient showed that the strains colonizing the same host, particularly those within cow, were slightly more homogenous (less binary distance and more correlated with low $D$-value) than those from the two hosts (i.e. considering cow and human strains together) (Fig. 3A ).

\section{Genotyping of ST239-MRSA clone}

Screening for mec A, van A and van B resistance genes revealed the presence of mec A gene in all examined ST239 strains confirming that they were all MRSA. Of note, two human and two animal isolates were identified as VRSA and they harbored van A orvan B genes.

Coagulase genotyping revealed that ST239-MRSA strains were genetically diverse and comprised a heterogeneous population with $D$-value equal to 0.79 (Fig. 4B ). Nine different coagulase genotypes $\left(\mathrm{C}^{\mathrm{I}}-\mathrm{C}^{\mathrm{IX}}\right)$ were detected (Fig. 4B ). The CI (band size $=750 \mathrm{bp}$ ) was the predominant coagulase type being identified in $38 \%$ of the strains $(19 / 50)$. Moreover, coa -RFLP was proven to be the best discriminatory method for investigating the ST239-MRSA strains as it gave rise to 19coa -RFLP patterns with high $D$-value of 0.92 (Fig. 4B, Table S2 ).

Concerning the SCCmec typing, ST239-MRSA strains were discriminated into five types $(D$-value $=0.46)$ with SCCmec -III being the most common one (72\%) (Table S3 ). The correlation between phenotypic resistance patterns and SCCmecgenotyping revealed a heterogeneous resistance profile of the strains belonged to SCCmec -III with $83.3 \%$ of them being MDR; meanwhile, all isolates of other SCCmec types were MDR (Fig. S1B ).

Grouping of agr alleles revealed only agr types I and III with $D$-value equal to 0.22 (Table S3 ). The agrtype I was the most prevalent $[88 \%,(44 / 50)]$. All SCCmectype III strains were of agr type I. interestingly, $86.4 \%(38 / 44)$ of the strains with agr type I were MDR (Fig. S1B ).

According to PCR amplification of spa-X region, the ST239-MRSA strains were grouped into 5 spa types with $D$-value equal to 0.53 . Majority of the strains $(64 \%, 32 / 50)$ were typed as $\mathrm{S} 1$ (400 bp). The S1 type was associated mainly with SCCmec -III andagr -I types being presented in $71.9 \%$ of the strains $(23 / 32)$ (Fig. 4B, Table S3 ).

\section{Toxin gene profiles}

Toxin gene profiles of ST239-MRSA strains revealed the presence of one or more toxin genes in $80 \%(40 / 50)$ of the tested strains. The most prevalent genes among the toxigenic strains were enterotoxins $(75 \%)$ and $p v l$ (57.5\%) (Fig.4B ). Our study indicated that the strains possessed only one type of the et genes. Of the nine enterotoxin genes examined, only sea, sec and seewere identified and all the toxigenic strains possessed only 
one type of these genes. The most prevalent enterotoxin gene among the examined strains was sea (40\%). The results also revealed thatpvl, see, sec and tst genes were more prevalent among the human strains compared to the animal ones, but the reverse occurred with sea, eta and etb genes. The combinative data of toxin gene profiles revealed 19 different toxin gene profiles with $D$-value equal to 0.91 (Fig. $4 \mathbf{B}$ ).

\section{Strains diversity based on their genotypic background among different hosts and sample types}

Depending on all genotyping methods, the analyzed ST239-MRSA strains showed a considerable overlap (Fig. 4A ) with no segregation into defined clusters with a high averaged binary distance reached 0.8 and a low average positive correlation coefficient $(\mathrm{R})$ of 0.2-0.3 among cow and human strains and between cow and human strains. The dissimilarity among strains of the same host was almost similar to that among different hosts (average binary distance $=0.78)($ Fig. 3C ), which holds true looking at each typing approaches separately.

Considering all genotyping approaches, the strains from the same sample were highly diverse, with the range of averaged binary distance between 0.6 and 0.8 and CSF isolates show the highest heterogeneity (average binary distance $=0.8$ and a very weak positive correlation $\mathrm{R}=0.03$ ) and the swab isolates constituted the least diverse pool (average binary distance $=0.6)($ Figure 3D ). The homogeneity of the ST2239-MRSA strains within each sample type and among different samples was quite similar, which was consistent for each of the genotyping approaches.

\section{Correlation among different typing methods}

Considering all ST239-MRSA isolates, the correlation analyses segregated all typing features into three main clusters (Fig. 2 ) and each cluster contained a pool of typing parameter, each belonged to different typing methods. The majority of the typing methods $(76.2 \%, 16 / 21)$ correlated to each other positively and only five of them correlated negatively. Finally, $98 \%$ of all ST239-MRSA strains have different genetic background when analyzed by combined genotyping methods (Fig. 4 ). Fortunately, all ST239-MRSA strains could be differentiated on the basis of their genetic background combined with their antimicrobial resistance profiles (Fig. S1B ). The coa -RFLP typing demonstrated the highest discriminatory power for strains within and among hosts in opposition to the agr typing.

\section{Correlation between the existence of antimicrobial resistances and toxin genes}

Our study also revealed a negative correlation between the existence of toxin genes and the antimicrobial resistances. Notably, MDR strains with maximum antimicrobial resistance patterns were associated with the least toxin gene profile. Overall, $50 \%$ of toxigenic strains showed low MAR indices ([?] 0.38), but higher MAR index ([?] 0.38) were observed in $80 \%$ of non-toxigenic strains (Fig. S1B ). All isolates derived from wound swabs were completely resistant to tetracycline, ceftriaxone and gentamicin and sensitive only to vancomycin in addition to their possession to $p v l$ gene (100\%). Furthermore, none of the isolates recovered from urine samples showed sensitivity to clindamycin and resistance to tetracycline, ceftriaxone, erythromycin, chloramphenicol, vancomycin,, trimethoprim-sulfamethoxazole and imipenem (Table 1 ).

\section{Correlation between genetic background and toxin genes}

The majority of Panton-Valentine leucocidin $(78.3 \%, 18 / 23)$, toxic shock syndrome toxin $(60 \%, 3 / 5)$, exfolative toxins $(83.3 \%, 10 / 12)$ and enterotoxins $(83.3 \%, 25 / 30)$ positive strains belonged to $a g r$-I genotype. Moreover, majority of the strains harboring enterotoxin genes $(56.7 \%, 17 / 30)$ were found to be belonged to SCCmec -III and 38.9\% (14/36) of ST239-MRSA-SCCmec -III strains possessedsea . Meanwhile, sec was found among ST239-MRSA SCCmec-II strains. The ST239-MRSA SCCmec-IV and -V were found to carry see and sea genes, respectively. Furthermore, $65.2 \%$ of $p v l, 58.33 \%$ of et and $40 \%$ oftst positive strains belonged to SCCmec -III. Interestingly, eta gene was distributed among ST239-MRSA SCCmec -III and $-\mathrm{V}$ and non-typeable strains, while etb gene was found among ST239-MRSA SCCmec -II and -III and non-typeable strains. All ST239-MRSA SCCmec -IV and -V and the majority of non-typeable strains had more than one toxin genes. Moreover, the majority of toxin producing ST239-MRSA strains belonged to spatype $1(57.5 \%, 23 / 40)$ and coa type I $(40 \%, 16 / 40)$ (Fig. 4B ). 


\section{Discussion}

The ST239-MRSA lineage is one of the most successful and persistent hybrid that is widely disseminated and causes numerous epidemics (Feil et al., 2008). Antimicrobial susceptibility testing of ST239-MRSA strains revealed the increasing prevalence of resistance to tetracycline, erythromycin, ciprofloxacin as was previously reported in China(Kong et al., 2017). The haphazard use of antimicrobials to treat bacterial infections in animals often increases the resistance to these antimicrobials. The adapted ability of ST239MRSA clone to enhanced antimicrobial resistance is extremely serious. Interestingly, more than $80 \%$ of our strains, mostly from human origin were MDR. This is consistent with a previous study conducted in India(Prashanth et al., 2011). Meanwhile, the MDR rate, especially among human strains in our study was higher than that previously reported in China(Yang et al., 2017). The indiscriminate use of antimicrobials in Egypt has rendered the commonly used antimicrobials completely ineffective in the treatment of this clone. In accordance with our study, some ST239-MRSA strains in Australia showed reduced vancomycin susceptibility (Holmes et al., 2014). So, there is a great challenge associated with the treatment of this clone.

Concerning the resistance to ciprofloxacin, clindamycin and gentamicin, human strains showed higher MAR indices than animal strains, while the reverse occurred with tetracycline, ceftriaxone and erythromycin. The continuous prescription of several Egyptian hospitals to ciprofloxacin, lincomycin and gentamicin in chest infections and the large applications of tetracycline and erythromycin in veterinary fields lead to the variations in the resistance patterns to those antimicrobials among human and animal strains. The coadministration of erythromycin and tetracycline in clinical field reflects the positive correlation between their resistances among our strains. Meanwhile, the differences in the mechanisms of resistance and genetic elements associated with the resistance to tetracycline and clindamycin(Fletcher and Macrina, 1991) may illustrate the negative correlation between these antimicrobials. The significant differences in the clindamycin resistance observed among our human and animal strains may be attributed to the absence of injection dosage form, which limits the veterinary use of this antimicrobial in Egypt in comparison with other antimicrobials.

The SCCmec molecular typing revealed that ST239-MRSA SCCmec III was the most prevalent clone in Egypt. In accordance with our study, lower proportions of diverse of SCCmec types II, IV and V and non-typeable ST239-MRSA strains were detected in previous studies(Chong et al., 2013).

Regarding the analysis of ST239-MRSA toxin gene profiles, our study indicated that nearly half of the strains were positive for pvlgene. This result contradicts previous studies (Figueiredo and Ferreira, 2014) suggesting that the carriage of $p v l$ gene may be unreliable marker for classifying the ST239-MRSA strains. Usually, the ST239-MRSA lineage lacks the tst gene, while few studies have reported the presence of this gene in ST239 strains (Iwao et al., 2012) as was presented in our finding, where only few strains carried tst gene. Notably, eta was determined to be the major exfoliative toxin type among our strains. This observation supports several researches in Europe, USA and Africa, where etawas harbored by more than $80 \%$ of toxigenic strains(Cribier et al., 1994). Thesea was the most common enterotoxin genes detected among MRSA strains (Tekeli et al., 2016). Similarly, our study showed that sea is the most prevalent enterotoxin gene among our ST239-MRSA strains.

Our study showed a positive correlation between the presence oftst gene and agr -I as the majority of TST producers belonged to agr -I. This is in disagreement with previous findings for isolation of toxic shock syndrome causing strains withagr -III allele(Bendary et al., 2016). In this report, we demonstrated that majority of ET producers belonged to agr -I. Previously, the majority of ETs producers belonged to agr -IV(Sheehan et al., 1992), while occasional strains belonging to the agr -I and -II also producing ETs(Jarraud et al., 2011). The strains with SCCmec -IV often carries pvlgene(Vandenesch et al., 2003). Nevertheless, our results agree with an earlier report(Liang et al., 2019), which confirmed the association of pvl positive strains to SCCmec -III. Herein, tst gene was proved to be associated with SCCmec -III. This is not in agreement with the previously reported predominance of tst gene among SCCmec-II strains(Liang et al., 2019). These observations support that there is no absolute correlation between toxin profiles and genetic background of this clone. 
In the current analyses, it was expected that the ST239-MRSA strains belonging to different hosts (i.e. humans and cows) would exhibit more divergence compared to that among strains of the same host, this was accepted phenotypically. Meanwhile, the diversity of both human and animal strains was almost similar to that among the same host depending on the genetic background. These results also suggest that the host is not the driving factor for the diversity of this clone, possibly ST239-MRSA strains within the same origins may have undergone different evolution processes. The great diversity of these strains provides a wide array of defense strategies and challenges to both prevention methods and therapies(Yamamoto et al., 2012).

Our study revealed an overall negative correlation between the existence of antimicrobial resistance and the toxin genes. The toxigenic ST239-MRSA clone had a non-MDR profile and they were susceptible to most antimicrobial agents, while MDR ST239-MRSA strains were less toxicogenic. This indicates the possibility of acquisition of toxin genes on mobile elements on the expense of extended antibiotic resistance and vice versa. The consequence of these practices among ST239-MRSA strains may provide a fortuitous opportunity to correlate phenotypic antibiotic resistance patterns with the occurrence of toxin genes(Beceiro et al., 2013).

\section{Conclusions}

The current study showed high diversity of ST239-MRSA clone, suggesting the low adaptability of such strains possibly due to their recombination or evolutionary dynamism. Most strain were MDR and belonged to SCCmec -III, agr -I, coa -I (750bp), spa -I (400bp) genotypes and harbored sea and pvl genes. These results have important implication, especially with regard to understanding the complex epidemiology of this clone in Egypt, which is ultimately linked to strategies of its prevention and control. Therefore, there is an urgent need for more specific recommendations as to the control and prevention of such pathogen as well as their resistance, in particular applying more restricted isolation guidelines within each host/hospital unit and also avoiding the abusing of antibiotics especially in veterinary fields. Future studies featuring large sample size are needed to reach solid conclusions regarding a more impacted infection control decisions.

\section{Acknowledgments}

We gratefully acknowledge all staff member in microbiology laboratory, Zagazig University for providing 30 ST239 MRSA strains and other human MRSA strains.

Funding: No funding or sponsorship was received for this study or publication of this article.

Conflicts of interest: The authors declare no conflict of interest.

\section{Author's contribution}

MB and MA designed the study, carried out the antibiotyping and molecular analyses and participated in data analyses. MS performed the Bioinformatics, statistical analyses and prepared the drafts and final version of the figures. MS, MB, MA and AS revised and discussed the figures, tables and the results. AS and AA wrote the primarily version of manuscript and participated in the design, antibiotyping, and molecular analyses. DG, WH, WA and RM conceived the study and participated in the analysis. HR reviewed and edited the manuscript. All authors have read and agreed to the published version of the manuscript.

\section{Data availability statement}

All data generated or analysed during this study are included in the published article or as supplementary information files. All sequences that have been generated on this article have been submitted to Genbank and will be available after (during) article publication

\section{References}

Abd El-Hamid, M. I. and M. M. Bendary, 2015: Comparative phenotypic and genotypic discrimination of methicillin resistant and susceptible Staphylococcus aureus in Egypt. Cell Mol Biol (Noisy-le-grand), 61, 101-112. 
Abd El-Hamid, M. I., M. M. Bendary, A. M. A. Merwad, I. Elsohaby, D. Mohammad Ghaith and W. A. Alshareef, 2019: What is behind phylogenetic analysis of hospital-, community- and livestock-associated methicillin-resistant Staphylococcus aureus? Transboundary and Emerging Diseases, 66, 1506-1517.

Baba T, T. F., Kuroda M, Yuzawa H, Aoki K, 2002: Genome and virulence determinants of high virulence community-acquired MRSA.. ;:. Lancet, 359, 1819-1827.

Beceiro, A., M. Tomás and G. Bou, 2013: Antimicrobial resistance and virulence: a successful or deleterious association in the bacterial world? Clin Microbiol Rev, 26, 185-230.

Becker, K., Skov, R. L., \& von Eiff, C., 2015: Staphylococcus, Micrococcus, and other catalase positive cocci. In J. H. Jorgensen, M. A. Pfaller, K. C. Carroll, G. Funke, M. L. Landry, S. S. Richter, D. W. Warnock (Ed.), Anonymous manual of clinical microbiology (11th ed., pp. 354-382). Washington, DC: American Society of Microbiology .

Bendary, M.M., S.M. Solyman, M.M. Azab, N.F. Mahmoud, A.M.Hanora, 2016: Characterization of Methicillin Resistant Staphylococcus aureus isolated from human and animal samples in Egypt. Cell. Mol. Biol, $62,94-100$.

Bendary, M.M., S.M. Solyman, M.M. Azab, N.F. Mahmoud, A.M.Hanora, 2016: Genetic diversity of multidrug resistant Staphylococcus aureus isolated from clinical and non clinical samples in Egypt. Cell. Mol. Biol , 62, 55 .

Chong, Y. P., E. S. Kim, S.-J. Park, K.-H. Park, T. Kim, M.-N. Kim, S.-H. Kim, S.-O. Lee, S.-H. Choi, J. H. Woo, J.-Y. Jeong and Y. S. Kim, 2013: Accessory gene regulator (agr) dysfunction in Staphylococcus aureus bloodstream isolates from South Korean patients. Antimicrob Agents Chemother, 57, 1509-1512.

CLSI, 2014: Methods for antimicrobial dilution and disk susceptibility testing of infrequently isolated or fastidious bacteria; approved guideline (M45-A). Wayne, PA: Clinical and Laboratory Standards Institute.

Cribier, B., Y. Piemont and E. Grosshans, 1994: Staphylococcal scalded skin syndrome in adults: A clinical review illustrated with a new case. Journal of the American Academy of Dermatology, 30, 319-324.

Enright, M. C., N. P. Day, C. E. Davies, S. J. Peacock and B. G. Spratt, 2000: Multilocus sequence typing for characterization of methicillin-resistant and methicillin-susceptible clones of Staphylococcus aureus. $J$ Clin Microbiol, 38, 1008-1015.

Feil, E. J., E. K. Nickerson, N. Chantratita, V. Wuthiekanun, P. Srisomang, R. Cousins, W. Pan, G. Zhang, B. Xu, N. P. J. Day and S. J. Peacock, 2008: Rapid detection of the pandemic methicillin-resistant Staphylococcus aureus clone ST 239, a dominant strain in Asian hospitals. J Clin Microbiol, 46, 1520-1522.

Figueiredo, A. M. and F. A. Ferreira, 2014: The multifaceted resources and microevolution of the successful human and animal pathogen methicillin-resistant Staphylococcus aureus.Memorias do Instituto Oswaldo Cruz, 109, 265-278.

Fletcher, H. M. and F. L. Macrina, 1991: Molecular survey of clindamycin and tetracycline resistance determinants in Bacteroides species. Antimicrob Agents Chemother,35, 2415-2418.

Franklin RC, Matthew AW, Jeff A, Michael ND and G. ME., 2012: Performance Standards for Antimicrobial Susceptibility Testing. Twenty-second Informational Supplement, M100-S22. U.S.A.: Wayne.

Friendly, M., 2002: Corrgrams. The American Statistician, 56, 316-324.

Galili, T., A. O'Callaghan, J. Sidi and C. Sievert, 2018: heatmaply: an R package for creating interactive cluster heatmaps for online publishing. Bioinformatics (Oxford, England), 34, 1600-1602.

Gilot, P., G. Lina, T. Cochard and B. Poutrel, 2002: Analysis of the genetic variability of genes encoding the RNA III-activating components Agr and TRAP in a population of Staphylococcus aureus strains isolated from cows with mastitis. J Clin Microbiol, 40, 4060-4067. 
Holmes, N., J. Turnidge, W. Munckhof, J. Robinson, T. Korman, M. O'Sullivan, T. Anderson, S. Roberts, S. Warren, G. Coombs, H.-L. Tan, W. Gao, P. Johnson and B. Howden, 2014: Genetic and Molecular Predictors of High Vancomycin MIC in Staphylococcus aureus Bacteremia Isolates. J Clin Microbiol, 52.

Hookey, J. V., J. F. Richardson and B. D. Cookson, 1998: Molecular typing of Staphylococcus aureus based on PCR restriction fragment length polymorphism and DNA sequence analysis of the coagulase gene. J Clin Microbiol, 36, 1083-1089.

Iwao, Y., O. Khokhlova, T. Takano, W.-C. Hung, H. Isobe, O. Peryanova, A. Salmina and T. Yamamoto, 2012: Fatal pneumonia in HIV-infected patients from a novel ST239 methicillin-resistant Staphylococcus aureus carrying the toxic shock syndrome toxin-1 gene in Krasnoyarsk, Siberian Russia. Japanese journal of infectious diseases, 65, 184-186.

Jarraud, S., G. J. Lyon, A. M. S. Figueiredo, G. Lina, F. Vandenesch, J. Etienne, T. W. Muir and R. P. Novick, 2011: Exfoliatin-producing strains define a fourth agr specificity group in Staphylococcus aureus $J$ Bacteriol, 193, 7027.

Jr, F. E. H., 2020: Package 'Hmisc'.https://cran.r-project.org/web/packages/Hmisc/Hmisc.pdf .

Kariyama, R., R. Mitsuhata, J. W. Chow, D. B. Clewell and H. Kumon, 2000: Simple and reliable multiplex PCR assay for surveillance isolates of vancomycin-resistant enterococci.J Clin Microbiol, 38, 3092-3095.

Katayama, Y., T. Ito and K. Hiramatsu, 2000: A new class of genetic element, staphylococcus cassette chromosome mec, encodes methicillin resistance in Staphylococcus aureus.Antimicrob Agents Chemother, 44, 1549-1555.

Kolde, -. R., 2018: Package 'pheatmap': pretty heat map

Kong, H., F. Yu, W. Zhang, X. Li and H. Wang, 2017: Molecular epidemiology and antibiotic resistance profiles of methicillin-resistant Staphylococcus aureus strains in a tertiary hospital in China. Frontiers in microbiology, 8, 838 .

Larsen, A., M. Stegger and M. Sorum, 2008: spa typing directly from a mecA, spa and pvl multiplex PCR assay - a cost-effective improvement for methicillin-resistant Staphylococcus aureus surveillance. Clinical Microbiology and Infection, 14, 611-614.

Liang, Y., C. Tu, C. Tan, M. Ahmed, M. Dai, Y. Xia, Y. Liu, L.-L. Zhong, C. Shen, G. Chen, G.-B. Tian, J. Liu and X. Zheng, 2019: Antimicrobial resistance, virulence genes profiling and molecular relatedness of methicillin-resistant Staphylococcus aureus strains isolated from hospitalized patients in Guangdong Province, China. Infection and Drug Resistance, 12, 447-459.

Magiorakos, A. P., A. Srinivasan, R. Carey, Y. Carmeli, M. Falagas, C. Giske, S. Harbarth, J. Hindler, G. Kahlmeter and B. Olsson-Liljequist, 2012: Multidrug-resistant, extensively drug-resistant and pandrugresistant bacteria: an international expert proposal for interim standard definitions for acquired resistance. Clinical microbiology and infection, 18, 268-281.

Mehrotra, M., G. Wang and W. M. Johnson, 2000: Multiplex PCR for detection of genes for Staphylococcus aureus enterotoxins, exfoliative toxins, toxic shock syndrome toxin 1 , and methicillin resistance. $J$ Clin Microbiol, 38, 1032-1035.

Monecke, S., G. Coombs, A. C. Shore, D. C. Coleman, P. Akpaka, M. Borg, H. Chow, M. Ip, L. Jatzwauk, D. Jonas, K. Kadlec, A. Kearns, F. Laurent, F. G. O'Brien, J. Pearson, A. Ruppelt, S. Schwarz, E. Scicluna, P. Slickers, H.-L. Tan, S. Weber and R. Ehricht, 2011: A field guide to pandemic, epidemic and sporadic clones of methicillin-resistant Staphylococcus aureus. PLoS One,6, e17936-e17936.

Prashanth, K., K. Rao, P. Vivek Reddy, R. Saranathan and A. Makki, 2011: Genotypic characterization of Staphylococcus aureus obtained from humans and bovine mastitis samples in India. Journal of Global Infectious Diseases, 3, 115-122. 
Robinson, D. A. and M. C. Enright, 2004: Evolution of Staphylococcus aureus by Large Chromosomal Replacements. J Bacteriol, 186, 1060.

Royston, P., 1982: An extension of Shapiro and Wilk's W test for normality to large samples. Applied Statistics, 31, 115-124.

Sheehan, B. J., T. J. Foster, C. J. Dorman, S. Park and G. S. A. B. Stewart, 1992: Osmotic and growth-phase dependent regulation of the eta gene of Staphylococcus aureus: a role for DNA supercoiling. Molecular and General Genetics MGG, 232, 49-57.

Simpson, E. H., 1949: Measurement of Diversity. Nature, 163, 688-688.

Tambekar, D., D. Dhanorkar, S. Gulhane, V. Khandelwal and M. Dudhane, 2006: Antibacterial susceptibility of some urinary tract pathogens to commonly used antibiotics. African Journal of Biotechnology, 5.

Team,-- R. C., 2018: R: A language and environment for statistical computing. R Foundation for Statistical Computing, Vienna, Austria,https://www.R-project.org/.

Tekeli, A., D. N. Ocal, B. B. Ozmen, Z. C. Karahan and I. Dolapci, 2016: Molecular Characterization of Methicillin-Resistant Staphylococcus aureus Bloodstream Isolates in a Turkish University Hospital Between 2002 and 2012. Microbial Drug Resistance, 22, 564-569.

Yamamoto, T., T. Takano, W. Higuchi, Y. Iwao, O. Singur, I. Reva, Y. Otsuka, T. Nakayashiki, H. Mori, G. Reva, V. Kuznetsov and V. Potapov, 2012: Comparative genomics and drug resistance of a geographic variant of ST239 methicillin-resistant Staphylococcus aureus emerged in Russia. PLoS One, 7, e29187.

Yang, X., S. Qian, K. Yao, L. Wang, Y. Liu, F. Dong, W. Song, J. Zhen, W. Zhou, H. Xu, H. Zheng and W. Li, 2017: Multiresistant ST59-SCCmec IV-t437 clone with strong biofilm-forming capacity was identified predominantly in MRSA isolated from Chinese children. BMC Infectious Diseases, 17.

Zhang, K., J.-A. McClure, S. Elsayed, T. Louie and J. M. Conly, 2005: Novel multiplex PCR assay for characterization and concomitant subtyping of staphylococcal cassette chromosome mec types I to $\mathrm{V}$ in methicillin-resistant Staphylococcus aureus. J Clin Microbiol, 43, 5026-5033.

Figure legends

Figure 1 : Distribution hierarchical clustering of ST239-MRSA strains the antimicrobial resistance variables based on the antimicrobial resistance profiles. A. Non-metric multidimensional scaling analysis showing the overlap of ST239-MRSA strains belonging to different hosts sample types. B. Heat map showing the occurrence of antimicrobial resistance in the studied ST239-MRSA strains. In the heat map red blue colors refer to resistance sensitivity to a particular antimicrobial respectively. The dendrogram represents the hierarchical clustering of the strains the antimicrobial resistance variables. The vertical lines dropped from the dendrogram branches show the 10 clusters that contained identical isolates. Different categories of hosts sample types are color-coded on the right of the heat map.

Figure 2: Pairwise correlation $(\mathrm{R})$ among different variables of typing methods. Red blue colors indicate positive negative correlation respectively. The color key refers to correlation coefficient (R). The darker colors imply stronger positive or negative correlations. Hierarchical clustering of the variables is shown as a dendrogram illustrating different clusters with different colors letters (e.g. A B C). Variables that are identical among all strains were excluded thus not shown in this figure.

Figure 3: Networks depicting the similarities dissimilarities among ST239-MRSA strains based on the binary distance. A. The average distance among strains from humans cows depending on their antimicrobial resistance profile B. The average distance among strains from various samples depending on their antimicrobial resistance profiles. C. The average distance among strains from humans cows depending on their genotyping profiles. B. The average distance among isolates from various samples depending on their genotyping profiles. 
Figure 4 : Distribution hierarchical clustering of ST239-MRSA strains the different genotypes based on different genotyping methods.A. Non-metric multidimensional scaling analysis showing the overlap of ST239-MRSA strains belonging to different hosts sample types. B. Heat map showing the occurrence of particular genotype variables in the studied ST239-MRSA strains. In the heat map red blue colors refer to presence absence of particular variables respectively. The dendrogram represents the hierarchical clustering of the strains the genotype variables. Different genotyping methods of hosts sample types are color-coded on the right of the heat map the genotyping categories are color-coded on the top of the heat map.

\section{Hosted file}

Tables 1.pdf available at https://authorea.com/users/361538/articles/482914-clonal-diversityand-epidemiological-characteristics-of-st239-mrsa-strains
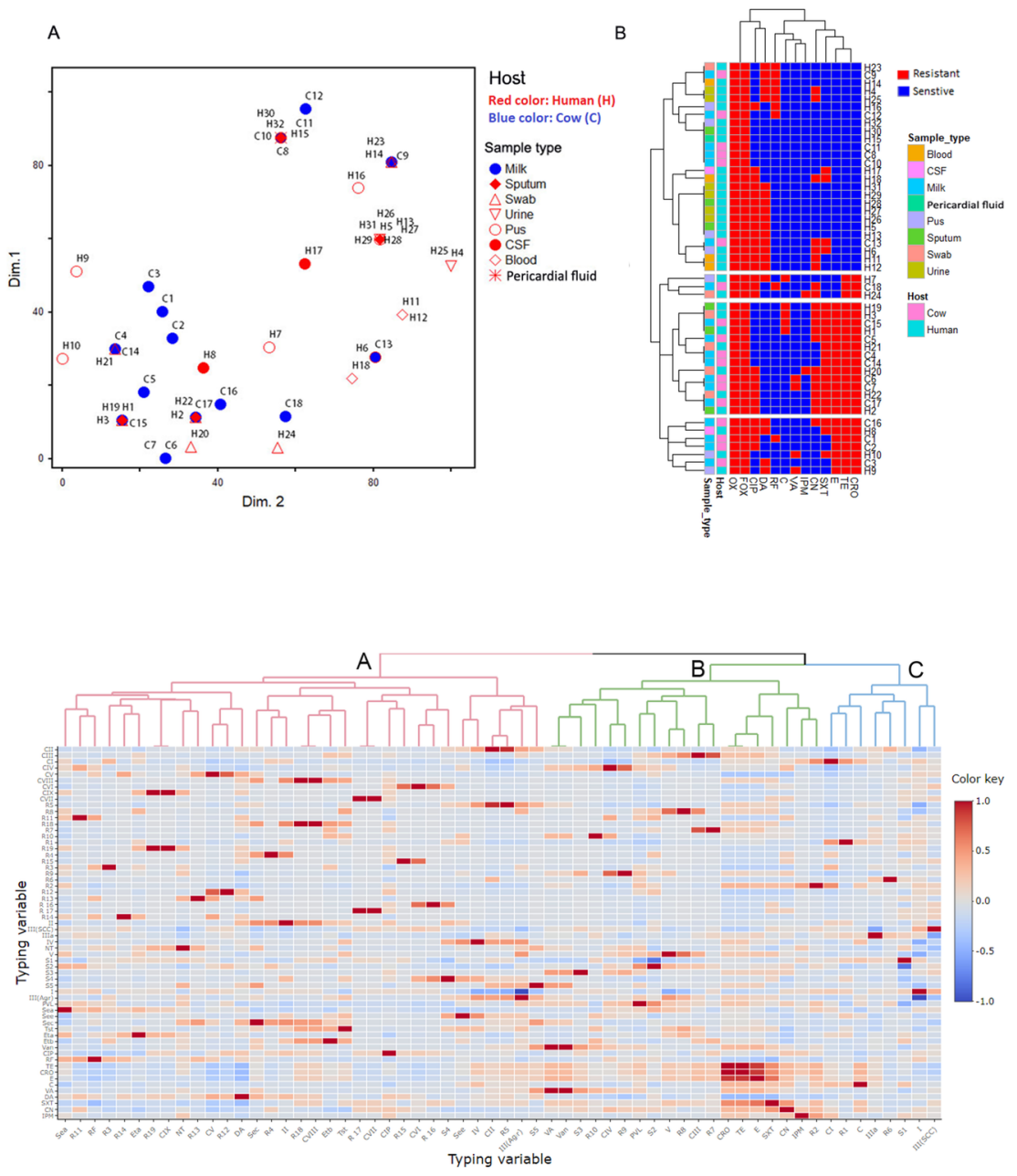

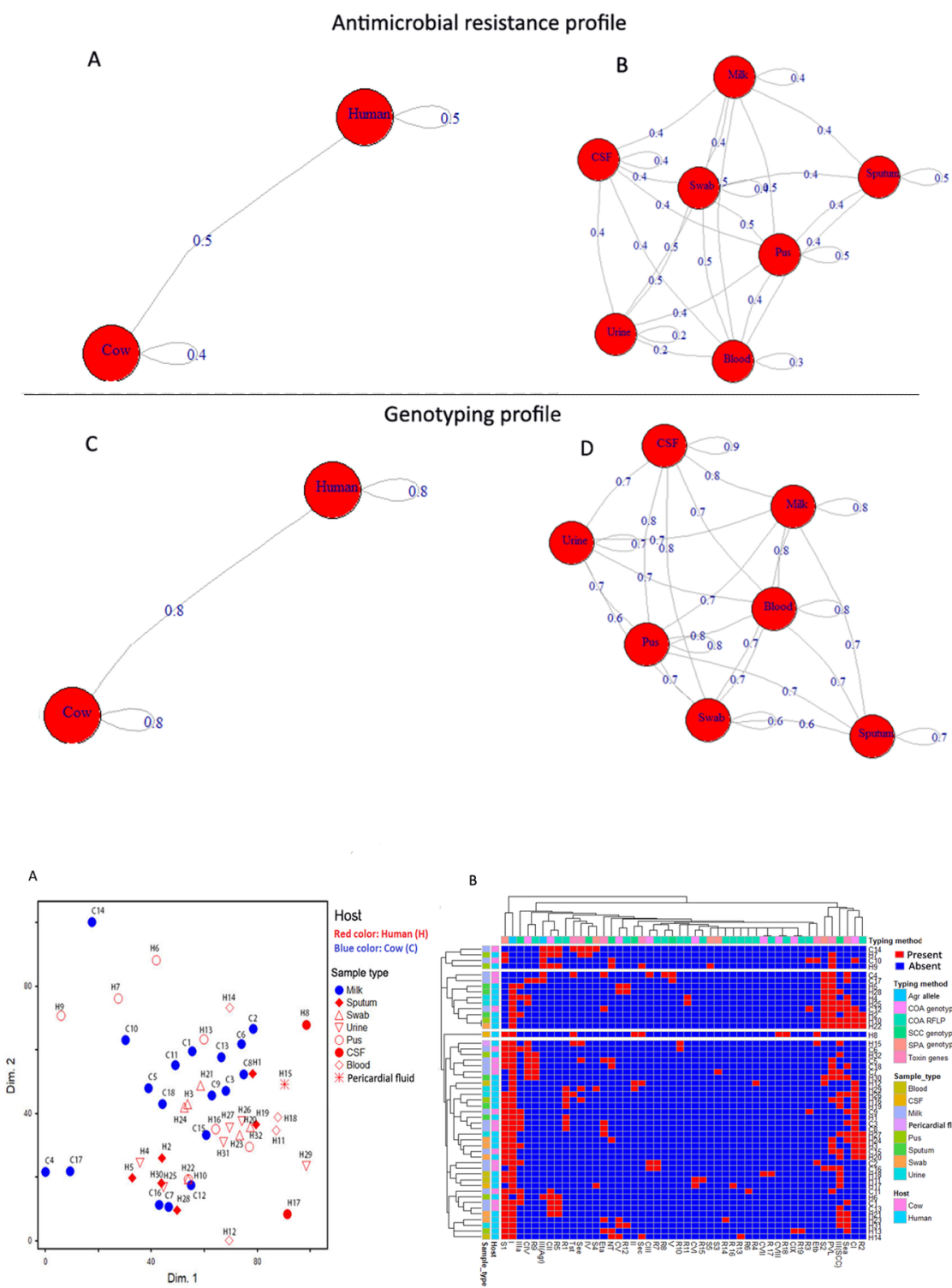

B

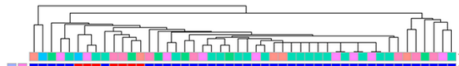

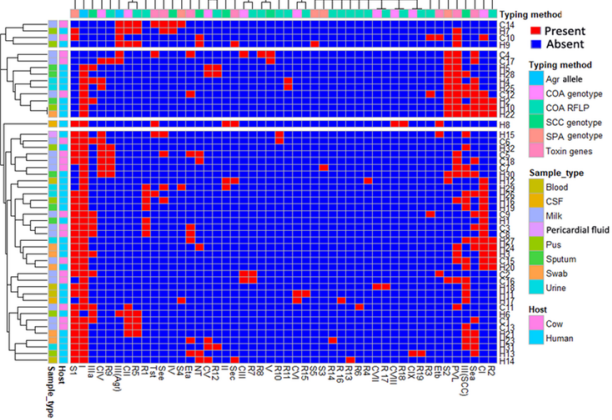

